\title{
Topology optimization of planar cross-sections
}

\author{
M. Chirehdast \\ Ford Motor Company, Advanced Vehicle Technology, 3632 SRL, P.O. Box 2053, Dearborn, MI 48121-2053, USA
}

\section{S.D. Ambo}

Department of MEAM, The University of Michigan, Ann Arbor, MI 48109, USA

\begin{abstract}
Topology optimization provides a rigorous method for the conceptual design of structural components. In this note, a practical approach for solving topology optimization problems of planar cross-sections is discussed. A problem formulation involving the use of continuous design variables is presented, and a standard nonlinear programming algorithm is used to solve the optimization problem. Results of the technique for two examples are presented and compared to similar results in the literature.
\end{abstract}

\section{Introduction}

Historically, structural component design has been a complex blend of experience, intuition, and heuristics. The advent of computers has fostered the widespread use of more rigorous optimization techniques for both conceptual and detailed phases of design. Structural optimization efforts generally are classified in three categories: (1) size optimization, which allows variations in the dimensions of a parameterized design, (2) shape optimization, which allows variations in the boundaries of a part while the overall layout remains unchanged, and (3) topology optimization where the layout, i.e. the connectivity of members, locations of holes, etc. are allowed to vary. Suzuki and Kikuchi (1991) provide a more complete overview of generalized shape and topology optimization.

This note aims to develop a procedure for determining optimal layouts for cross-sections. The motivation for designing optimal cross-sections is widespread. However, the automotive industry has considerable interest, particularly in the area of joint design. Constraints on geometry, section properties and manufacturing with minimum weight usually as a driving factor require extensive efforts to determine a suitable, if not optimal, cross-section.

In the past two decades, several researchers have studied shape optimization methods that deal specifically with structural cross-section design. Hou and Chen (1985) presented a method to find optimal cross-sectional shapes of elastic hollow bars. Schramm and Pilkey (1993) illustrate a $B$-spline representation of the cross-section to optimize a bar in torsion.

Recently, the use of discrete optimization techniques to solve topology optimization problems was proposed. Chapman et al. (1993) used a genetic algorithm to develop twodimensional configurations. Sandgren et al. (1990) also applied a genetic optimization algorithm to determine topologies for three-dimensional shell components. Another ap- proach taken by Anagnostou et al. (1992) employs simulated annealing to solve topology optimization problems both in structural design and heat transfer.

In the general area of topology optimization, Suzuki and Kikuchi (1991) applied the homogenization method to solve generalized layout problems for two- and three-dimensional components. The resulting designs have minimum compliance for a specified constraint on material volume. Similar solutions have been obtained by Rozvany et al. (1992) using the solid isotropic microstructure with the penalty (SIMP) method.

The remainder of this note is organized as follows. In Section 2, the problem formulation used to solve topology optimization problems of cross-sections is described. In Section 3, two examples of cross-section optimization using this approach are presented, followed by conclusions and future work in Section 4.

\section{Formulation}

In order to numerically solve a topology optimization problem, commonly the design space or domain is discretized into finite elements. The formulation used here for the crosssection problem does not differ in this regard. In genetic algorithms and simulated annealing methods, a binary representation for each element is used. Every element either contains solid material or is completely void.

However, the approach taken here allows material accumulation in each element to continuously vary from void to solid. As shown in Fig, 1, each element contains a rectangular hole of arbitrary size at the centroid of the element. The sizes of the hole (denoted by $a$ and $b$ ) serve as design variables in the formulation of the optimization problem. Without loss of generality, the elements may be considered to be of unit height and width.

For all of the applications presented in this note, the hole is assumed to be square, i.e. $a=b$, although the method is applicable for the general variable definition. Given these assumptions, the following element properties can be defined in terms of $a$ and $b$ : cross-sectional area, moment of inertia about the $x$-axis, and moment of inertia about the $y$-axis. An area moment of inertia may be calculated with respect to a global coordinate system using the Parallel Axis Theorem. The contributions of each element can be summed to obtain the overall moment of inertia. 


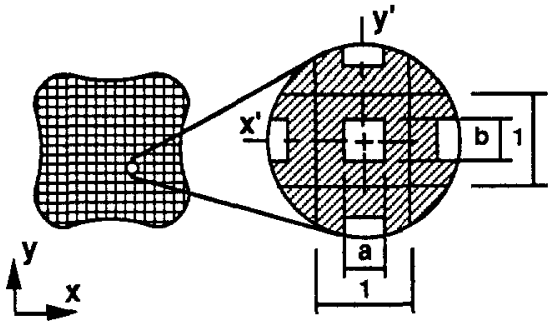

Fig. 1. An arbitrary design domain discretized into elements with rectangular holes

Additional section properties such as centroid location, shear centre location, etc. can be analytically expressed with respect to this model if desired. Packaging limitations on the cross-sectional geometry are intrinsically implemented when creating the finite-element model. Generally, in cross-section design the goal is to maximize a section property or minimize weight. The examples that follow are weight (area) minimization problems with constraints on particular performance criteria, such as the ones mentioned above.

The formulation as presented does not ensure symmetric designs. Forcing symmetry through variable linking can be implemented easily and is often avoided by using appropriate modelling techniques. However, a more critical issue is to ensure that the layout is connected. Currently, there is no provision for avoiding unconnected designs; this limitation remains an open issue. One advantage of using a genetic algorithm, or other discrete optimization methods, is that unconnected designs can be discarded.

\section{Examples}

One of the more common cross-sections used in structures is the $I$-beam, which has good bending stiffness characteristics. A reasonable problem formulation for the design of an $I$-beam would be:

$\min A$ s.t. $\quad \bar{I}_{x}-I_{x} \leq 0$,

where $A$ and $I_{x}$ are the area and the area moment of inertia of the cross-section, respectively, and $\bar{I}_{x}$ is an active lower bound on the latter.

We applied the method described above to solve this particular problem. The model consisted of 100 square elements of unit area, with one design variable per element. Several nonlinear programming codes such as GRG2 (Lasdon et al. 1978), VMCON (Crane et al. 1980), and NLPQL (Schittkowski 1984) were used to solve the optimization problem. The same solution was found with these codes as shown in Fig. 2.

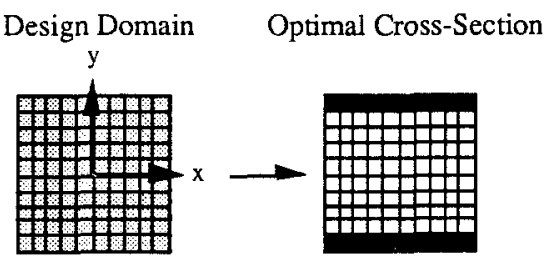

Fig. 2. Initial design with even distribution of material and final distribution of material

Note that the results strongly indicate a cross-section design consisting of two wide flanges farthest from the neutral axis. Since the problem formulation does not prohibit unconnected designs, the result does not exhibit the centre web typical of $I$-beams.

A similar problem was investigated by Chapman et al. (1993) using a genetic algorithm. The problem was formulated to maximize the strength to weight ratio of a crosssection in a rectangular domain subject to no constraints. Using this ratio as an objective function and utilizing a continuous variable approach, we found the results to indicate a thin flange design as in Fig. 2. However, additional optimization using a finer mesh shows that the solution is dependent on the mesh discretization. Analytically, it can be proven that the fitness function attains its maximum at the limit as the flange thickness goes to zero.

A more complicated example involves placing a second constraint on the area moment of inertia about the $y$-axis; a similar problem has been solved by Anagnostou et al. (1992). The goal in this example is to design a cross-section similar to the square box beam shown below. The values of the moments of inertia about the $x$-and $y$-axis of the box beam are used as constraints in the optimization. Only one quarter of the section is analysed due to the symmetry of the problem. The model consists of 900 elements and the solution shown in Fig. 3 was obtained with the sequential quadratic programming-based codes mentioned previously (VMCON and NLPQL). GRG2 was not able to robustly find the optimum. NLPQL was the most efficient algorithm and converged to the same solution from several different starting points. The area of the optimized cross-section is 34.5 versus 36.0 for the box beam section.

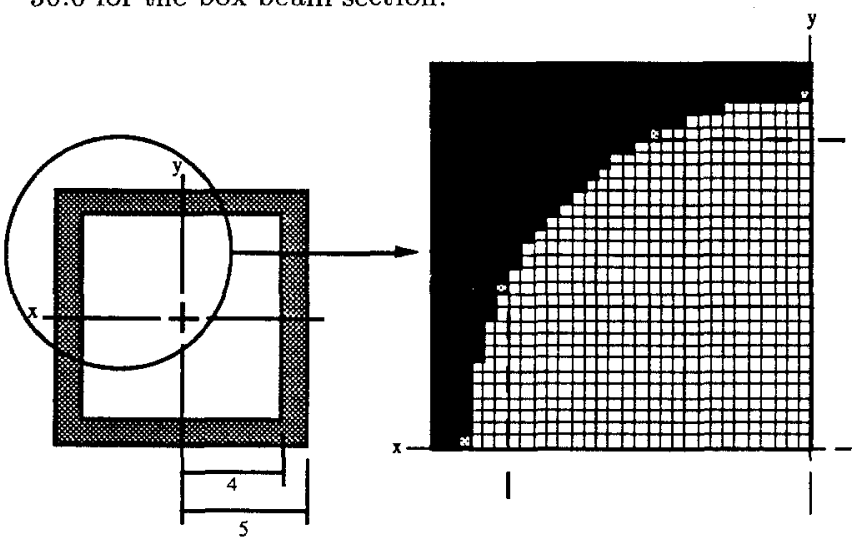

Fig. 3. Concept box beam and optimized cross-section $(1 / 4$ model)

The results suggest that a cross-section with a circular hole in the centre is lighter than a cross-section of equivalent moment of inertia properties with a square hole. Analytically, it can be proven that the cross-sectional area is minimized when the number of sides of a regular $n$-sided polygon inside the square cross-section reaches infinity, i.e. the hole becomes circular, see the Appendix for an explicit proof.

\section{Conclusions}

A continuous variable formulation and standard nonlinear programming algorithms are presented as a viable approach for solving general topology optimization problems of planar cross-sections. However, one limitation of the proposed 
approach is the potential for unconnected designs. In this note, weight minimization problems with constraints on section properties are solved. For this case, NLPQL was found to be the most efficient and robust algorithm that can handle a large number of design variables in real time. The next immediate extension of this study is to apply the approach to torsional rigidity problems.

\section{References}

Anagnostou, G.; Ronquist, E.M.; Patera, A.T. 1992: A computational procedure for part design. Comp. Meth. Appl. Mech. Eng. 97, $33-48$

Chapman, D.; Saitou, K.; Jakiela, M 1993: Genetic algorithms as an approach to configuration and topology design. Proc. 1993 ASME Design Automation Conf. pp. 1-14

Crane, R.L.; Hillstrom, K.E.; Minkoff, M. 1980: Solution of the general nonlinear programming problem with subroutine VMCON. Report ANL-80-64, Argonne National Laboratory, Mlinois

Hou, J.W.; Chen, J.L. 1985: Shape optimization of elastic hollow bars. Trans. ACM 107, 100-105

Lasdon, L.S.; Warren, A.D.; Jain, A.; Ratner, M. 1978: Design and testing of a generalized reduced gradient code for nonlinear programming. ACM Trans. Mathematical Software, pp. 35-50

Rozvany, G.I.N.; Zhou, M.; Birker, T. 1992: Generalized shape optimization without homogenization. Struct. Optim. 4, 250-252

Sandgren, E.; Jensen, E.; Welton, J. 1990: Topological design of structural components using genetic optimization methods. In: Sensitivity analysis and optimization with numerical methods, 115, pp. 31-43. ASME

Received April 6, 1994
Schittkowski, K. 1984: Design, implementation, and test of a nonlinear programming algorithm. Report, Institut für Informatik, Stuttgart University

Schramm, U.; Pilkey, W.D. 1992: Structural shape optimization for the torsion problem using direct integration and B-splines. Comp. Meth. Appl. Mech. Eng. 107, 251-268

Suzuki, K.; Kikuchi, N. 1991: A homogenization method for shape and topology optimization. Comp. Meth. Appl. Eng. 93, 291-318

\section{Appendix}

The problem of area minimization of a square cross-section (of size $a$ ) with a hole that is a regular $n$-sided polygon ( $n=$ $2 \pi / \phi$ ) can be formulated as follows:

$\min a^{2}-\frac{2 \pi}{\phi} R_{1}^{2} \tan (\phi)$,

subject to $I \leq \frac{\pi}{12 \phi} R_{1}^{4} \tan (\phi)\left[\frac{6-4 \sin ^{2}(\phi)}{\cos ^{2}(\phi)}\right]$,

where $R_{1}$ is the radius of the inscribing circle of the polygon and $I$ is a lower bound on the area moment of inertia of the cross-section. The same problem can be formulated as the following unconstrained problem:

$\max \frac{2 \pi}{\phi} \tan (\phi) \sqrt{\frac{12 \phi I \cos ^{2}(\phi)}{\pi \tan (\phi)\left[6-4 \sin ^{2}(\phi)\right]}}$,

which has its solution at $\phi=0$ or as the number of sides of the polygon goes to infinity. The first, second and third derivatives of the unconstrained function vanish, and the fourth derivative is negative (equals $-\frac{16}{15} \sqrt{2 \pi I}$ ) indicating a local maximum. 